\title{
MORTALIDADE POR NEOPLASIAS DOS ÓRGÃOS GENITAIS MASCULINOS: DESAFIOS NA PREVENÇÃO E DETECÇÃO PRECOCE
}

\author{
MORTALITY FROM NEOPLASMS OF MALE GENITAL ORGANS: \\ CHALLENGES IN PREVENTION AND EARLY DETECTION
}

Andreza Silva Costa ${ }^{1}$

Tais Layane de Sousa Lima²

Bruna Braga Dantas ${ }^{3}$

RESUMO: Objetivo: Analisar a mortalidade por neoplasias malignas dos Órgãos Genitais (OG) masculinos no Brasil, no período de 1999 á 2018, identificando os principais desafios na prevenção e detecção precoce, buscando contribuir com a integração dos homens nas práticas preventivas. Metodologia: Estudo documentaldescritivo, considerando duas décadas: 1999-2008 e 2009-2018, no Brasil, por meio de duas plataformas: Sistema de Informações sobre Mortalidade- SIM, em que se coletaram os óbitos por neoplasias dos órgão genitais masculinos, e o Atlas de Mortalidade Online- INCA, no qual se coletou a taxa ajustada de mortes pelas neoplasias de próstata, pênis e testículos. Utilizou-se também, o auxílio da literatura disponível sobre a temática abordada. Resultados: Nas décadas analisadas, 1999-2008 e 2009-2018, a mortalidade das Neoplasias dos Órgãos Genitais (NOG) masculinos foi de 11,92 e 15,79 para cada 100 mil homens, respectivamente. A mortalidade por câncer de próstata destacou-se entre as demais, com 10,55 e 14,36 mortes para cada 100 mil homens, nas respectivas décadas. O déficit de autocuidado, acarretado por idealizações culturais e sociais masculinas, levam a baixa adesão às práticas preventivas, a atenção em saúde estar mais voltada para o processo de cura, quando se trata do públicomasculino. Conclusão: Conclui-se que a mortalidade por neoplasias dos OG masculinos aumentou no período estudado, com destaque para a mortalidade por câncer de próstata. Identificou-se que as principais dificuldades na prevenção e detecção precoce dessas patologias estão

\footnotetext{
1 Graduanda do curso de Enfermagem da Universidade Federal de Campina Grande, Campus Cuité. andrezasssilva8@gmail.com.

2 Graduanda do curso de Enfermagem da Universidade Federal de Campina Grande, Campus Cuité. thaislayane1817@gmail.com.

${ }^{3}$ Doutora em Enfermagem, docente da Universidade Federal de Campina Grande, Campus Cuité. bruna.braga@professor.ufcg.edu.br.
} 
relacionadas aos ideais de masculinidade, déficit de autocuidado e atenção a saúde masculina pautada no curativismo.

Palavras chave: Neoplasias. Sistema reprodutor. Saúde do homem.

ABSTRACT: Objective: To analyze the mortality from malignant neoplasms of the male Genital Organs (OG) in Brazil, from 1999 to 2018, identifying the main challenges in prevention and early detection, seeking to contribute to the integration of men in preventive practices. Methodology: Documentary-descriptive study, consideringtwo decades: 1999-2008 and 2009-2018, in Brazil, through two platforms: Mortality Information System - YES, in which deaths from neoplasms of the male genital organs were collected, and the Online Mortality Atlas - INCA, which collected the adjusted rate of deaths from prostate, penis and testicular neoplasms. It was also used the help of the available literature on the topic addressed. Results: In the analyzed decades, 1999- 2008 and 2009-2018, the mortality of male Genital Organ Neoplasms (NOG) was 11.92 and 15.79 for every 100 thousand men, respectively. Mortality from prostate cancer stood out among the others, with 10.55 and 14.36 deaths for every 100 thousand men, in the respective decades. The deficit in selfcare, caused by male cultural and social ideals, leads to low adherence to preventive practices, health care being more focused on the healing process, when it comes to the male audience. Conclusion: It is concluded that the mortality from neoplasms of the male OG increased during the studied period, with emphasis on the mortality from prostate cancer. It was identified that the main difficulties in the prevention and early detection of these pathologies are related to the ideals of masculinity, self-care deficit and attention to male health based on curativism.

Keywords: Neoplasms; Reproductive system; Men's Health. 


\section{INTRODUÇÃO}

O organismo humano é composto por uma junção de sistemas, a exemplo do sistema reprodutor, que tem por finalidade executar o processo de reprodução da espécie. Existem dois sistemas reprodutores na espécie humana, o feminino e o masculino, o último é responsável por formar, expelir e inserir nos canais que compõemo sistema reprodutor feminino, um líquido denominado esperma ou sêmen, podendo resultar assim na fecundação. O sistema reprodutor masculino é formado por diversos órgãos, alguns deles são: pênis, testículos e próstata, que são nomeados de Órgãos Genitais (OG) (DANGELO, FATTINI, 2007). Assim como os demais órgãos, os OG também são formados por tecidos, estruturas compostas pela união de células que podem sofrer alterações em seu DNA, e essas mutações podem resultar em uma transformação maligna, originando um câncer, comprometendo assim o tecido e consequentemente o órgão, dando origem às neoplasias dos órgãos genitais (NOG) (INCA, 2020).

O câncer, também denominado neoplasia maligna, enquadra-se como um dos problemas mais sérios nos quais o sistema de saúde brasileiro presta assistência, devido a sua relevância nos âmbitos epidemiológicos, sociais e econômicos. As neoplasias caracterizam-se como uma multiplicação incomum das células, em que o organismo perde o controle da função local de modo parcial ou total, o que favorece a autonomia e continuidade dessa proliferação, com implicações severas sobre o hospedeiro. As neoplasias malignas, também denominadas de tumores malignos, apresentam uma manifestação com maior nível de autonomia, tendo à capacidade de adentrar em tecidos próximos, provocando desse modo a metástase, comumente, resistentes ao tratamento, podendo provocar o óbito do indivíduo acometido (INCA, 2020; OLIVEIRA et al., 2019).

Segundo o INCA (2020), um terço dos casos novos de câncer no mundo poderia ser evitado, porém, no que tange ao câncer de próstata, a prevenção colide em grande parte das vezes com a deficiência de atenção à saúde do homem, 
devido a idealizações culturais e sociais próprias do público masculino. O Instituto Nacional do Câncer elenca como principais fatores causais da doença, os riscos ambientais, ocupacionais, geralmente ligados ao exercício profissional e também os fatores genéticos, que podem predispor o indivíduo ao câncer.

O diagnóstico precoce das neoplasias é extremamente relevante, visto que quanto antes ocorrer à detecção do câncer e consequentemente a implantação do referido tratamento, maior será a probabilidade de um bom prognóstico, possibilitando assim, uma intervenção mais eficiente e com maior possibilidade de cura, logo, resultando em uma melhor qualidade de vida para o paciente. Desse modo, é imprescindível que os profissionais de saúde compreendam os sinais, sintomas e fatores de risco das neoplasias, pois a realização de um diagnóstico precoce contribuiria para um bom prognóstico (STEFFEN et al., 2018).

Nesse contexto, considerando os pontos de vista cultural e social, a atenção à saúde permanece sendo assimilada como uma prática voltada para o público feminino, e que dá prioridade as variadas fragilidades extremadas pelas esferas sociais e seus processos de saúde e doença. Embora as organizações de saúde tenham objetivado trabalhar os serviços de saúde com base na universalidade, integridade e equidade, o imaginário representativo ainda reitera a prática do cuidado como zona feminina, suprimindo desse modo a extensão do masculino (PNAISH, 2008).

A adesão do público masculino às práticas de saúde preventiva tornou-se uma considerável dificuldade tanto para as Políticas Públicas quanto para os profissionais de saúde, visto que os homens correspondem a índices de morbimortalidade mais altos em comparação às mulheres, demonstrando que a presença dos mesmos nos serviços de atenção primária à saúde é menos significativa que a participação feminina (MARTINS et al., 2019). Usualmente, os indivíduos do sexo masculino não têm o reconhecimento das particularidades inerentes a sua saúde, e não são inclusos nas populações normalmente acompanhadas pelos serviços prestados pelas unidades de atenção básica àsaúde (BIONDO et al., 2020).

Assim, buscando assegurar a promoção do aperfeiçoamento das condições de saúde para o público masculino do Brasil, foi criada a Política Nacional de 
Atenção Integral à Saúde do Homem (PNAISH), que possui como um dos seus eixos temáticos oacesso e acolhimento, na busca de promover uma reorganização das ações de saúde por intermédio de uma orientação inclusiva, visando que os serviços de saúde reconheçam que os indivíduos do sexo masculino precisam de cuidados específicos, evidenciando assim que os serviços de saúde também são espaços masculinos (PEREIRA; KLEIN;MEYER, 2019).

É inegável que a política nacional de atenção à saúde do homem forma um relevante método de promoção da saúde, no entanto ainda se faz necessário que os profissionais da saúde passem a analisar com mais atenção os fatores que afastam os homens das unidades de saúde, pois é evidente que esse público possui mais dificuldades quando se trata de autocuidado, e a conduta adotada pelos profissionais frente a isso, pode proporcionar melhores resultados no que se refere à prevenção de doenças e promoção de saúde para esse público específico (VAZ et al., 2018).

Diante dos fatos abordados, o referido estudo tem como finalidade analisar a mortalidade por neoplasias malignas do sistema reprodutor masculino no Brasil, no período de 1999 á 2018, e identificar os principais desafios na prevenção e detecção precoce dessas neoplasias, buscando contribuir assim, com a integração do público masculino nas práticas preventivas voltadas à essas patologias.

\section{MATERIAIS E MÉTODO}

Foi realizado um estudo documental-descritivo, considerando duas décadas: 1999 á 2008 e 2009 á 2018, com dados obtidos por meio de duas plataformas: o Sistema de Informações sobre Mortalidade- SIM, em que se coletou o número de óbitos por neoplasias dos órgãos genitais masculinos, e o Atlas de Mortalidade Online- INCA, no qual se coletou a taxa ajustada de mortes pelas neoplasias de próstata, pênis e testículos, ambas as coletas consideraram a população brasileira. Posteriormente, foi realizado um cálculo para converter o número de óbitos por neoplasias dos órgãos genitais em taxa ajustada, considerando as décadas de 1999 
á 2008 e 2009 á 2018, ajustadas pelas populações brasileiras de 2000 e 2010, respectivamente, e esses dados foram comparados, buscando observar a existência de aumento ou redução da mortalidade no intervalo entre as duas décadas. Utilizouse também, o auxílio da literatura disponível sobre a temática abordada, por meio das palavras-chaves: Saúde do Homem, Prevenção e Detecção Precoce.

\section{RESULTADOS E DISCUSSÕES}

Quando se refere às neoplasias, torna-se evidente que muitas dessas são silenciosas em seus estágios iniciais, não promovendo sinais ou sintomas claramente visíveis, o que induz a falsa sensação de ausência de doença, desse modo, as ações preventivas em saúde são reconhecidas como fatores importantes e decisivos na detecção precoce dessas patologias, ao passo que pode vir a possibilitar um melhor prognóstico para o paciente, aumentando as probabilidades de cura, porém, a efetivação desse importante fator torna-se um problema, caso não consigam alcançar a população alvo de suas ações, a exemplo do público masculino (STEFFEN et al., 2018).

Os homens são menos adeptos a consultas médicas, o que caracteriza um déficit de autocuidado com a saúde, isso é acarretando por aprendizados culturais, e a não adesão às boas práticas de saúde está, por diversas vezes, vinculada a conceitos de ser uma fragilidade o homem buscar atendimento em saúde, e isso não faria parte do ideal de masculinidade cultivado. Essa postura conduz aos diagnósticos tardios, possibilitando maus prognósticos e reduzindo a expectativa de vida desse público (MOURA; RABELO, 2019).

As consequências de diagnósticos tardios, principalmente, tratando-se de neoplasias malignas, tornam-se evidentes ao analisar dados de mortalidade devido a essas doenças, visto que o diagnóstico precoce eleva a probabilidade de um bom prognóstico e amplia as chances de cura. De acordo com a figura 1, constatou-se que nas décadas analisadas, 1999 á 2008 e 2009 á 2018, a mortalidade por NOG masculinos foi de 11,92 e 15,79 para cada 100 mil homens, respectivamente, assim, 
evidenciando- se um aumento expressivo no intervalo de duas décadas, nesse contexto, torna-se visível à necessidade do fortalecimento das práticas preventivas na saúde do homem.

Figura 1. Mortalidade por neoplasias dos órgãos genitais masculinos considerando os períodos de 1999 á 2008 e 2009 á 2018, ajustada pelas populações de 2000 e 2010, respectivamente, no Brasil.

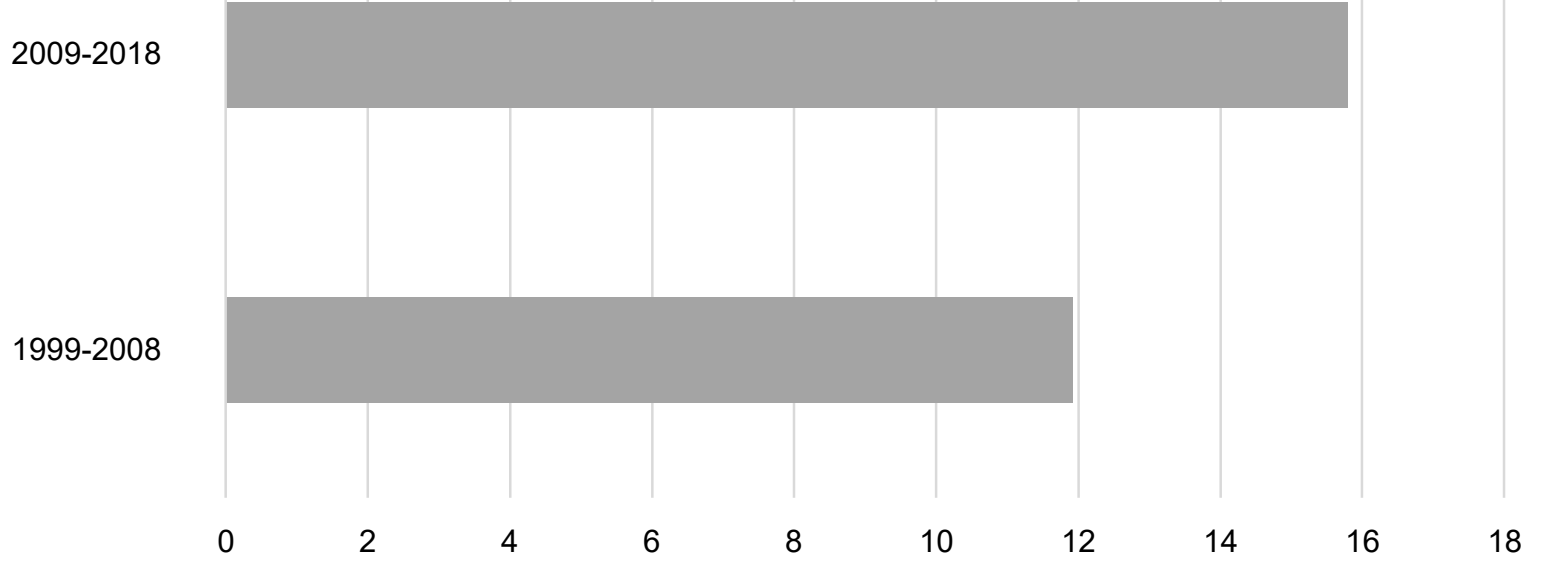

Fonte: Banco de dados do estudo, 2021.

Diante dos relevantes resultados expostos anteriormente, julgou-se pertinente analisar também, a mortalidade por neoplasias de alguns órgãos masculinos específicos, que geralmente são alvo do câncer com maior frequência, são eles: próstata; testículos e pênis.

Segundo a figura 2, que considera as neoplasias de próstata, testículos e pênis, percebem-se consideráveis aumentos de mortalidade no intervalo de duas décadas, para os três órgãos analisados. Dentre essas neoplasias, a que obteve maior taxa de mortalidade dentre o público masculino foi as neoplasia prostática, com 10,55 mortes para cada1 00 mil homens no primeiro intervalo temporal (19992008) e 14,36 mortes para cada 100 mil homens no segundo intervalo (2009-2018), em seguida, em ordem decrescente, estão o câncer de pênis e testículos. 
Figura 2. Taxa de mortalidade por neoplasias de próstata, pênis e testículos, para cada100 mil homens, nos períodos de 1999-2008 e 2009-2018, no Brasil.

\begin{tabular}{ccc}
\hline NEOPLASIA & $1999-2008$ & $2009-2018$ \\
\hline PRÓSTATA & 10,55 & 14,36 \\
\hline PÊNIS & 0,27 & 0,39 \\
\hline TESTÍ́CULOS & 0,25 & 0,33
\end{tabular}

Fonte: Banco de dados do estudo, 2021.

Os dados expostos nos permitem observar que houve consideráveis aumentos na mortalidade pelas neoplasias abordadas no intervalo de 20 anos, destacando assim, a importância do diagnóstico precoce, que é um determinante fundamental para um bom prognóstico, possibilitando mais chances de cura e uma melhor qualidade de vida para esse paciente, refletindo assim nos dados de mortalidade, no entanto, ainda é visível a persistência de estigmas em torno dos exames que possibilitam a detecção dessa patologia em estágio inicial.

A ascensão durante os vinte anos de descentralização do departamento de saúde,o SUS (Sistema Único de Saúde), ainda está em estágio de aperfeiçoamento, sendo necessário que as barreiras sejam desfeitas para que haja um funcionamento eficaz, de acordo com os princípios e diretrizes pré-estabelecidos. Uma dessas barreiras é o baixo índice de indivíduos do sexo masculino que buscam as unidades de atenção básica à saúde, preferindo assistência da atenção ambulatorial e hospitalar, de média lalta complexidade (SANTANA et al., 2011; KRUNGER, CAVALCANTI, 2018).

A atenção das politicas de saúde está mais voltada ao processo de cura de patologias quando se trata do público masculino, desenvolvendo menos práticas de prevenção e promoção de saúde, caracterizando uma das possíveis dificuldades na identificação precoce das NOG masculino, assim deve-se considerar também, que os homens apresentam dificuldade em aderir às práticas preventivas relacionadas a essas neoplasias, posto que, por diversas vezes são invasivas, e mexem com o imaginário masculino gerando constrangimento individual e social, devido aos conceitos de masculinidade trabalhados ao longo da sua vida (VAZ et al., 2018). 
O conceito que caracteriza os serviços prestados pela atenção primaria à saúde como direcionados quase que unicamente para o público feminino, infantil e idoso é muito difundido. Existem diversas hipóteses e até justificativas em relação à reduzida participação dos homens nas Unidades Básicas de Saúde (UBS). Relaciona-se o distanciamento dos homens nesses referidos serviços, a um aspecto inerente à identidade dos indivíduos desse sexo, que está associada a sua formação social. Nesse sentido, essa identidade estaria ligada a ideia da não valorização das práticas de autocuidado (PENA et al., 2018). Frente a isso, é notório que os indivíduos do sexo masculino vivenciam aprendizados desfavoráveis quanto à prevenção e promoção na saúde do homem, o que dificulta a adesão a essas práticas.

Os homens são habitualmente enxergados como símbolos de força, os que por sua vez os tornariam invencíveis, assim, procuram assistência apenas quando se encontram em situações graves, quando o problema passa a afetar seu desempenho no trabalho, por exemplo. Esses aspectos estão associados ao conceito de sexualidade masculina idealizado na sociedade. Assim, a formação da sexualidade masculina pode vir a ser influenciada pelo modelo do senso comum quanto à comprovação de ser efetivamente homem (QUIRINO et al., 2017; MODESTO et al., 2018).

Alguns métodos para integrar o público masculino nas ações preventivas podem ser desenvolvidos por profissionais de saúde, com o objetivo de mudar esse cenário, sensibilizando o publico masculino quanto à importância de aderir às práticas preventivas ofertadas pela atenção primária em saúde. A sensibilização pode ser iniciada por meio da identificação dos fatores que ocasionam a morbimortalidade dentre esse público, juntamente a situação social, econômica e cultural dos homens que serão alvo das ações de saúde (OLIVEIRA et al., 2019).

O público masculino terá que ser incluído no planejamento das ações que são ofertadas, tornando-se assim peça chave para o desenvolvimento das ações. Os profissionais de saúde, que lidam com indivíduos do sexo masculino, precisam ampliar a utilização de dinâmicas a serem realizas em grupos pequenos, favorecendo a troca de diálogo entre os participantes, levando assim à reflexão dos integrantes. As dinâmicas podem ser utilizadas para promover uma discussão 
quanto aos problemas de saúde que afetam os homens, a exemplo das neoplasias do sistema reprodutor masculino, nessa discussão deve-se permitir que os participantes se envolvessem, compartilhando ideias e situações vivenciadas por eles (DUARTE, OLIVEIRA, SOUZA, 2012).

Portanto, é de grande relevância fortalecer e ampliar os métodos de educação em saúde, estimular e atentar a população masculina quanto à adesão a um estilo de vida saudável e da importância de ir às instituições de saúde realizar práticas preventivas, com foco principal nos cuidados ofertados pela atenção primária. Além disso, é imprescindível analisar métodos de encaixar os profissionais de saúde em locais onde existe uma grande concentração de homens habitualmente, a exemplo de ambientes de trabalho, locais onde se podem utilizar estratégias de educação em saúde, como a entrega de panfletos informativos, palestras, organização de oficinas, orientações em grupo e até avaliações quanto ao risco de desenvolver determinadas doenças, como as neoplasias.

\section{CONSIDERAÇÕES FINAIS}

Em virtude do que foi exposto, observou-se que a mortalidade por neoplasias dos órgãos genitais masculinos aumentou no intervalo das duas décadas estudadas, obtendo destaque a mortalidade por câncer de próstata, que se encontrou superior em comparação ao câncer de pênis e testículos, considerando o mesmo período de tempo. Nesse contexto, identificou-se que as principais dificuldades que interferem na prevenção e detecção precoce das neoplasias dos órgãos genitais masculinos são, principalmente, os ideais de masculinidade ainda presentes na conjuntura social, que enxergam os cuidados preventivos em saúde como sinal de fragilidade, que seria incompatível com o conceito de masculinidade, e a priorização das unidades de atenção primária em realizar mais ações voltadas para as mulheres, crianças e idosos, reduzindo a atenção à saúde do homem.

Frente a esses aspectos, existem métodos que podem vir a facilitar a abordagem da equipe de saúde ao público masculino, integrando-os nas práticas de 
prevenção e detecção precoce das NOG masculinos. As ações de educação em saúde são um meio de programar e implementar os métodos de abordagem, inserindo profissionais de saúde nos locais com maior concentração de homens, realizando palestras, avaliação de risco, dinâmicas em grupo, dentre outras ações que visam informar e sensibilizar os homens quanto à importância da adesão aos métodos preventivos das referentes neoplasias, levando-os a procurar com mais frequência os serviços da atenção primária para realizar consultas preventivas, elevando assim as chances de obter o diagnóstico precoce dessas neoplasias, aumentando as chances de cura e consequentemente reduzindo as taxas de mortalidade.

\section{REFERÊNCIAS BIBLIOGRÁFICAS}

BIONDO, C. S. et al. Detecção precoce do câncer de próstata: atuação de equipe desaúde da família. Enfermería Actual de Costa Rica, n. 38, p. 32-44, 2020.

BRASIL. Ministério da Saúde. Saúde do homem. Glossário Temático. $1^{\text {a }}$ edição -2018 versão eletrônica. Brasília - DF 2018. Disponível em: https://pt.slideshare.net/Marcusrenato/sade-do-homem-glossrio-temtico-ministrio-da- sade. Acesso em: 15 nov. 2020. BRASIL. Ministério da Saúde, 2020. Banco de dados do Sistema Único de Saúde - DATASUS. Informações de Saúde, Sistema de Informações sobre Mortalidade. Disponível em: http://www.datasus.gov.br/catalogo/sim.htm. Acesso em: 28 out. 2020.

BRASIL. MINISTÉRIO DA SAÚDE. Política Nacional de Atenção Integral à Saúde doHomem PNAISH. Princípios e Diretrizes, 2008. Brasília: MS.

DANGELO, J. G.; FATTINI, C. A. Anatomia humana básica. 3. Ed. São Paulo: Atheneu, 2007.

DUARTE, S. J. H., OLIVEIRA, J. R., SOUZA, R. R. A política saúde do homem e sua operacionalização na atenção primária à saúde. Revista Eletrônica Gestão \& Saúde • Vol.03, №. 01, Ano 2012 p. 308-317.

INCA. Instituto Nacional do Câncer. Câncer no sistema reprodutor masculino. Rio de Janeiro, 2020. Disponível em: https://www.inca.gov.br. Acesso em: 17 out. 2020.

KRUGER, F.P.G.; CAVALCANTI, G. Conhecimento e atitudes sobre o cancer de próstata no Brasil: Revisão Integrativa. Revista Brasileira de Cacerologia, v.64, n.4, p.561-567, 2018.

MARTINS, E. R. C., et al. Vulnerabilidade de homens jovens e suas necessidades de saúde. Esc. Anna Nery. vol.24 no.1 Rio de Janeiro 2020 Epub Jan 13, 2020.

MOURA, F. V. M; RABELO, J.B. Aspectos Socioculturais que envolvem o Câncer de Próstata na Ótica dos Usuários e Assistentes Sociais. Revista Brasileira de Cancerologia, v.65, n.2, p.1-11, 2019.

OLIVEIRA, P. S. D. et al. Câncer de Próstata: conhecimentos e interferências na promoção e 
prevenção da doença. Enfermería Global, n.54, p.262-273, 2019.

OLIVEIRA, L. B. O., et al. Histologia dos tecidos. 1. ed., Pelotas Rio Grande do Sul: Pelotas, 2019.

PENA, C. S. et al. O papel da enfermagem mediante a resistência do homem naprevenção do câncer de próstata. Revista Científica Univiçosa, v.10, n.1, p. 269-274, Viçosa-MG, Jan./ Dez. 2018.

PEREIRA, J., KLEIN, C., MEYER, D.E. Uma análise de sua dimensão educativa na perspectiva de gênero. Rev. Saúde Soc. São Paulo, v.28, n.2, p.132-146, 2019.

QUIRINO, A.F.A. et al. O tabu masculino relacionado a prevenção do câncer de próstata. Revista Mundo Saúde e Biológicas, v.2, n.1, p. 1-22, Curitiba-PR, Jan./ Jul. 2017.

SANTANA, E. N., et al. A atenção à saúde do homem: ações e perspectivas dos enfermeiros. REME - Rev. Min. Enferm.;15(3): 324-332, jul./set., 2011.

STEFFEN, R. E. et al. Rastreamento populacional para o câncer de próstata: mais riscosque benefícios. Physis: Revista de Saúde Coletiva, v. 28, p. e280209, 2018.

VAZ, C. A. M. et al. Contribuições do enfermeiro para a saúde do homem na atenção básica. Revista de Iniciação Científica e Extensão, [S. I.], v. 1, n. 2, p. 122-126, 2018. 